\title{
Interference-Aware OFDMA Resource Allocation: A Predictive Approach
}

\author{
Stefan Geirhofer and Lang Tong \\ School of Electrical and Computer Engineering \\ Cornell University, Ithaca, NY 14853 \\ Email: $\{$ sg355, 1t35\}@ cornell.edu
}

\author{
Brian M. Sadler \\ Army Research Laboratory \\ Adelphi, MD 20783-1197 \\ Email: bsadler@arl.army.mil
}

\begin{abstract}
As wireless systems continue to proliferate, interference management is becoming a concern in both military and commercial domains. This paper introduces a novel cognitive coexistence framework between infrastructure and adhoc networks. Based on sensing and predicting the ad-hoc network's activity, the infrastructure system allocates power and transmission time such as to minimize its impact on the ad-hoc links. This leads to an interference-aware resource allocation. A rate-constraint ensures that the infrastructure system maintains a specified quality-of-service, despite adapting its transmission behavior to accommodate ad-hoc users.

Based on an ON/OFF continuous-time Markov chain model, the optimal allocation of power and transmission time is formulated as a convex optimization problem. Closed-form solutions are derived as a function of Lagrange multipliers. An iterative algorithm with guaranteed convergence to the optimal solution is developed. Finally, our results are extended to an averagerate formulation. Numerical performance analysis illustrates that utilizing the superior flexibility of the infrastructure links can effectively mitigate interference.
\end{abstract}

Index Terms-Cognitive Radio; Radio Resource Management; Standards Coexistence; Dynamic Spectrum Access;

\section{INTRODUCTION}

The growing demand in wireless services has led to a dense spectrum allocation, which makes it difficult to accommodate emerging standards in dedicated frequency bands. Interference management is therefore becoming increasingly important as spectrum needs to be shared among heterogeneous systems. Cognitive radio (CR) presents a possible solution by promoting techniques that adapt their transmission behavior dynamically to avoid interference.

In licensed bands, CR can be used to reuse underutilized frequency bands while protecting incumbent users from interference, an approach that is referred to as dynamic spectrum access (DSA). See [1], [2] for surveys on this topic. In unlicensed bands, where all radios have equal access rights, the same CR techniques may be used opportunistically to enable coexistence and reduce interference. The focus of this paper is to analyze a special instance of such cognitive coexistence methods.

\footnotetext{
${ }^{1}$ This paper was prepared though collaborative participation in the Communications and Networks Consortium sponsored by the U.S. Army Research Laboratory under the Collaborative Technology Alliance Program, Cooperative Agreement DAAD19-01-2-0011. The U.S. Government is authorized to reproduce and distribute reprints for Government purposes notwithstanding any copyright notation thereon.
}

Within this framework we address the coexistence of infrastructure (IS) and ad-hoc (AH) networks as depicted in Fig. 1. Motivated by the fact that IS systems typically possess superior computation and communication resources, we propose a cognitive resource management protocol that, based on sensing and predicting the activity of the $\mathrm{AH}$ network, allocates its power and transmission time to reduce interference. Despite adapting its behavior based on the $\mathrm{AH}$ system, a rate constraint ensures that the IS link maintains a specified quality-of-service level.

This approach differs from typical DSA setups in which a secondary system adapts its transmission behavior subject to not interfering with primary users [1]. Instead of imposing an interference constraint we consider adapting the primary's (IS system's) behavior subject to not reducing its quality-ofservice. This leads to "best-effort" interference mitigation for the secondary (AH system).

The proposed framework applies to several practical scenarios. In the military domain, the coexistence of infrastructure links (e.g., communication with a central command) and shortrange communication systems (e.g., sensor networks) is of fundamental importance [3]. In the commerical domain, similar challenges are faced in enabling license-exempt WiMAX operation [4], [5] or supporting peer-to-peer traffic in broadband cellular networks [6].

\section{A. Main contribution}

The coexistence of IS and AH wireless links is addressed within a cognitive framework. Based on sensing and predicting the behavior of the AH system, the superior flexibility of the IS links is used to allocate resources such that interference is reduced. Based on an OFDMA physical-layer, this is accomplished by finding a power and time allocation that has the smallest (expected) overlap with the AH links subject to providing a specified rate (quality-of-service) across the IS link.

The optimal power and time allocation, based on per-frame sensing results, is obtained by deriving the structure of the optimal solutions. Closed-form expressions for both power and time allocation are obtained as a function of a set of Lagrange multipliers. An algorithm for finding the optimal values of these Lagrange multipliers with guaranteed convergence is provided based on the bisection method. The per-frame allo- 
cation problem is extended to an average formulation in which the long-term statistics of the ad-hoc links are known.

\section{B. Related work}

Resource allocation in multi-user multicarrier networks has received much attention. In the absence of interfering networks the optimal sub-channel and power allocation has been wellstudied for both downlink and uplink scenarios [7], [8], [9]. In cognitive radio networks, resource allocation typically aims at maximizing the cognitive system's throughput subject to additional interference constraints, which protect the primary system [10], [11]. Contributions on optimal spectrum sharing and self-coexistence among secondaries include [12], [13], [14], [15].

The problem of allocating transmission time in an OFDMA system to improve coexistence has received little attention. To the best of the authors' knowledge, the optimal allocation of transmission time based on interference prediction has not been addressed before.

Within the framework of cognitive coexistence we have previously studied the optimum power allocation of IS and $\mathrm{AH}$ networks assuming knowledge of the interference channel [16]. The coexistence of local and personal area networks has been addressed in [17], [18] based on Bluetooth/WLAN coexistence.

\section{Problem Formulation}

This section introduces the system setup and formulates the proposed interference-aware resource allocation mathematically. This leads to a convex optimization problem, whose optimal solutions will be derived in subsequent sections.

\section{A. System setup}

Consider the system setup depicted in Fig. 1, where a single IS link coexists with a set of AH nodes. The IS uplink transmissions may cause significant interference to AH transmissions (downlink IS transmissions are assumed out-of-band and not considered in this paper). We study the problem of reducing mutual interference by judiciously allocating power and transmission time of the IS system.

Ad-hoc system: The AH system evolves continuously in time based on a decentralized and distributed medium access protocol. Its cumulative ON/OFF behavior is modeled based on a two-state continuous time Markov chain (CTMC) $X(t)$ with transition rates $\mu$ and $\lambda$ in the ON and OFF state, respectively [17]. The problem can be easily extended to multiple ad-hoc links that operate in parallel frequency bands (as shown in Fig. 2).

Infrastructure links: The IS system evolves in frames of fixed duration $T$ and uses an OFDMA physical-layer with $N$ sub-channels as depicted in Fig. 2. For simplicity we focus on a single IS client and its link to the base station. Our results are, however, readily extended to the case of multiple clients as long as these are served on orthogonal sets of sub-channels.

Based on detecting the activity of the $\mathrm{AH}$ network at the beginning of every frame, power and transmission time are

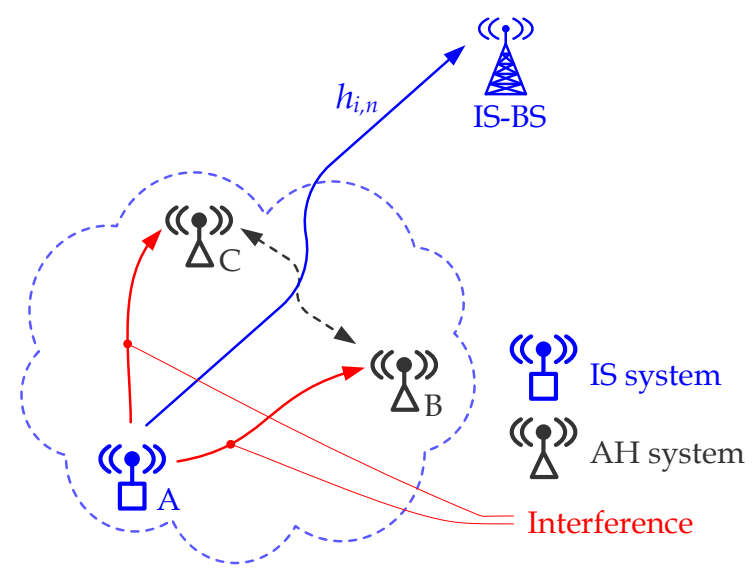

Fig. 1. System setup. An infrastructure link allocates its transmission time and power such as to create minimum interference to close-by ad-hoc networks.

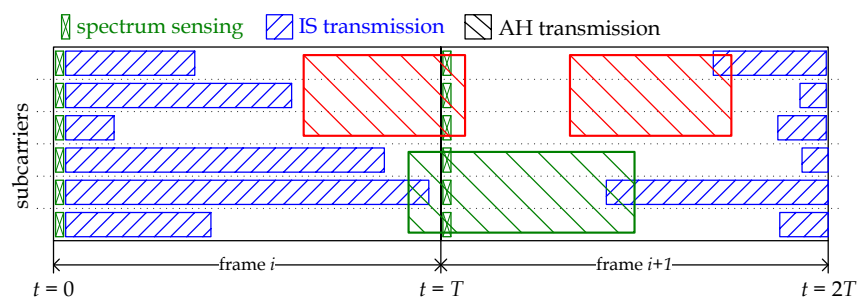

Fig. 2. OFDMA physical layer of the infrastructure system. A set of sub-channels overlaps with the ad-hoc network. Through channel sensing at the beginning of every frame, an interference-aware resource allocation is achieved.

allocated per sub-channel. Let $\mathbf{p}=\left[p_{1}, \ldots, p_{N}\right]^{T}$ denote the power allocation and $\boldsymbol{\rho}=\left[\rho_{1}, \ldots, \rho_{N}\right]^{T}$ the transmission time allocation $\left(\rho_{i}\right.$ is the fraction of the frame duration for which we use sub-channel $i$ ).

Periodic spectrum sensing at the beginning of every frame is the foundation of the proposed method. We assume that the IS client senses whether the AH system is currently active (ON or OFF) but that no information on the interference channel is available. For simplicity we assume that the sensing results can be obtained perfectly without any overhead.

Resource allocation is performed subject to the constraint that the IS link maintains a specified rate requirement $R$. Based on the power allocation $\mathbf{p}$ and transmission time allocation $\rho$, the sum rate of the IS link is given by

$$
\sum_{n} \rho_{n} \log \left(1+\kappa \frac{p_{n}\left|h_{n}\right|^{2}}{\rho_{n} N_{0}}\right)=\sum_{n} \rho_{n} \log \left(1+\frac{p_{n} \beta_{n}}{\rho_{n}}\right),
$$

where $N_{0}$ denotes the noise power, $\kappa$ is a normalization factor, and $\beta_{n}$ is introduced for notational convenience ${ }^{1}$.

\section{B. Interference metrics}

The proposed method aims at minimizing the average interference between IS and $\mathrm{AH}$ links. This requires us to

\footnotetext{
${ }^{1}$ The above formulation encompasses a channel capacity formulation (for $\kappa=1)$ as well as the case of variable-rate M-QAM in which case $\kappa=$ $1.5 /(-\ln B E R)$ is chosen such that a target BER is met [19].
} 
find the expected time overlap between transmissions of both systems which will form the cost function that is minimized by the allocation procedure.

Based on the CTMC model of the AH link, its transition matrix is given by [20, p.391]

$$
\mathbf{P}(t)=\frac{1}{\lambda+\mu}\left[\begin{array}{ll}
\mu+\lambda e^{-(\lambda+\mu) t} & \lambda-\lambda e^{-(\lambda+\mu) t} \\
\mu-\mu e^{-(\lambda+\mu) t} & \lambda+\mu e^{-(\lambda+\mu) t}
\end{array}\right] .
$$

If the $\mathrm{AH}$ system is observed in state $x$ at time $\tau$ through spectrum sensing, $[\mathbf{P}]_{(x, y)}$ is the probability of it being in state $y$ at time $\tau+t$. If the chain is ergodic, which we will assume hereafter, it is well-known that these probabilities converge to the stationary distribution in a monotonic fashion [20]. This property leads to the following lemma.

Lemma 1: Assume that a fraction $\rho_{n}$ of transmission time needs to be allocated to a sub-channel $n$, whose ON/OFF behavior is modeled by the CTMC (2). Based on a sensing outcome at the beginning of the frame, the minimal expected overlap is achieved by

- transmitting at the beginning of the frame (i.e., during $\left.\left[0, \rho_{n} T\right]\right)$ if the sensing outcome was idle and

- transmitting at the end of the frame (i.e., during [(1 $\left.\left.\rho_{n}\right) T, T\right]$ ) if the sensing outcome was busy.

Proof: This is an immediate consequence of the monotonicity of (2).

Define the expected overlap (normalized by $T$ ) as $\phi_{x}\left(\rho_{n}\right)$, where $x \in\{0,1\}$ denotes the sensing outcome ( $x=1$ denotes busy and $x=0$ represents idle). In the idle case it is given by

$$
\begin{aligned}
& \phi_{0}\left(\rho_{n}\right)=\frac{1}{T} \int_{0}^{\rho_{n} T} \operatorname{Pr}(X(\xi)=1 \mid X(0)=0) d \xi \\
& =\frac{\lambda}{(\lambda+\mu) T}\left(\rho_{n} T+\frac{1}{\lambda+\mu}\left(e^{-(\lambda+\mu) \rho_{n} T}-1\right)\right),
\end{aligned}
$$

where we used the transition matrix (2). In the case of a busy sensing outcome we obtain similarly,

$$
\begin{aligned}
& \phi_{1}\left(\rho_{n}\right)=\frac{1}{T} \int_{\left(1-\rho_{n}\right) T}^{T} \operatorname{Pr}(X(\xi)=1 \mid X(0)=1) d \xi \\
= & \frac{\lambda}{(\lambda+\mu) T}\left(\rho_{n} T+\frac{\mu / \lambda}{\lambda+\mu} e^{-(\lambda+\mu) T}\left(e^{(\lambda+\mu) \rho_{n} T}-1\right)\right) .
\end{aligned}
$$

Lemma 2: The functions $\phi_{0}\left(\rho_{n}\right)$ and $\phi_{1}\left(\rho_{n}\right)$ are strictly convex and increasing in $\rho_{n}$.

Proof: Both $\phi_{0}\left(\rho_{n}\right)$ and $\phi_{1}\left(\rho_{n}\right)$ are nonnegative linear combinations of a convex and a strictly convex function. One is linear, the other an exponential function with nonzero exponent. The monotonicity can be easily verified by differentiation.

\section{Minimizing interference temperature}

Based on the functions $\phi_{0}\left(\rho_{n}\right)$ and $\phi_{1}\left(\rho_{n}\right)$, the problem of minimizing the interference power which may impact the $\mathrm{AH}$ links can be formulated as (given sensing outcome $x$ )

$$
\begin{aligned}
\min _{\mathbf{p}, \boldsymbol{\rho}} & \sum_{n} \frac{p_{n}}{\rho_{n}} \phi_{x}\left(\rho_{n}\right) \\
\text { s.t. } & r(\mathbf{p}, \boldsymbol{\rho})=\sum_{n} \rho_{n} \log \left(1+\frac{p_{n} \beta_{n}}{\rho_{n}}\right) \geq R \\
& \sum_{n} p_{n} \leq P \\
& p_{n} \geq 0, \quad \forall n \\
& 0 \leq \rho_{n} \leq 1, \quad \forall n
\end{aligned}
$$

where the objective function represents the expected transmit power that overlaps with transmissions of the AH system. Note that $p_{n}$ denotes the total transmit power that is allocated to a sub-channel (the peak power is therefore given by $p_{n} / \rho_{n}$ ). In addition to the rate constraint (6), there is a total power constraint (7).

The above optimization problem is not convex since the objective function is not convex in $[\mathbf{p}, \boldsymbol{\rho}]$. As a result (5)(9) is difficult to solve. We therefore consider a convex approximation which lends itself to a more tractable solution.

\section{Minimizing time overlap}

The complication in (5)-(9) arises from the fact that the product $p_{n} \phi_{x}\left(\rho_{n}\right)$ is not convex in $\left[p_{n}, \rho_{n}\right]$. We therefore consider an approximation to the above problem which is based on the observation that due to the relatively high transmit power of the IS link, the transmission overlap primarily affects the amount of interference. This leads to the formulation (again, based on sensing outcome $x$ )

$$
\begin{aligned}
\min _{\mathbf{p}, \boldsymbol{\rho}} & \sum_{n} \phi_{x}\left(\rho_{n}\right) \\
\text { s.t. } & r(\mathbf{p}, \boldsymbol{\rho})=\sum_{n} \rho_{n} \log \left(1+\frac{p_{n} \beta_{n}}{\rho_{n}}\right) \geq R \\
& \sum_{n} p_{n} \leq P \\
& p_{n} \geq 0, \quad \forall n \\
& 0 \leq \rho_{n} \leq 1, \quad \forall n,
\end{aligned}
$$

which minimizes the expected overlap between both systems, subject to the same constraints as before. Note that the power allocation $\mathrm{p}$ has been eliminated only from the objective function. It is still an important factor in leveraging channel diversity based on knowledge of the channel coefficients $\beta_{n}$.

The expressions (10)-(14) form a convex optimization problem, since the objective function is convex in $\rho$, the rate constraint is convex in $[\mathbf{p}, \boldsymbol{\rho}]$ (by the perspective function property [21]), and all other functions are linear in the decision variables.

Although convex programs can be solved using general solution techniques, we will further exploit the special structure of this problem to arrive at a low-complexity solution. 


\section{Optimal Resource Allocation}

The paper develops the optimal resource allocation as a solution to the convex optimization problem (10)-(14). Based on the optimality conditions, closed-form expressions for $\mathbf{p}$ and $\rho$ are obtained as a function of Lagrange multipliers. An iterative algorithm based on the bisection method is used to find the optimal values for these Lagrange multipliers.

\section{A. Optimality conditions and solution structure}

The Lagrangian of the convex program is obtained by introducing Lagrange multipliers $\gamma \geq 0$ and $\epsilon \geq 0$ for the rate and power constraint, respectively. This leads to

$$
\begin{aligned}
& L(\mathbf{p}, \boldsymbol{\rho} ; \gamma, \epsilon)=\sum_{n} \phi_{x}\left(\rho_{n}\right)+ \\
& \gamma\left[R-\sum_{n} \rho_{n} \log \left(1+\frac{p_{n} \beta_{n}}{\rho_{n}}\right)\right]+\epsilon\left[\sum_{n} p_{n}-P\right] .
\end{aligned}
$$

The regularity conditions $\mathbf{p} \geq 0$ and $0 \leq \boldsymbol{\rho} \leq 1$ have not been included into the Lagrangian but will be absorbed directly into the optimality conditions.

The Karush-Kuhn-Tucker (KKT) optimality conditions are given by the set of constraints (11)-(14), non-negativity constraints for the Lagrange multipliers, $\gamma \geq 0$ and $\epsilon \geq 0$, the slackness conditions

$$
\begin{aligned}
\gamma\left[R-\sum_{n} \rho_{n}^{*} \log \left(1+\frac{p_{n}^{*} \beta_{n}}{\rho_{n}^{*}}\right)\right] & =0 \\
\epsilon\left[\sum_{n} p_{n}^{*}-P\right] & =0
\end{aligned}
$$

the condition

$$
\left.\frac{\partial L(\mathbf{p}, \boldsymbol{\rho} ; \gamma, \epsilon)}{\partial p_{n}}\right|_{p_{n}=p_{n}^{*}}\left\{\begin{array}{cc}
=0, & p_{n}^{*}>0 \\
>0, & p_{n}^{*}=0
\end{array}\right.
$$

and

$$
\left.\frac{\partial L(\mathbf{p}, \boldsymbol{\rho} ; \gamma, \epsilon)}{\partial \rho_{n}}\right|_{\rho_{n}=\rho_{n}^{*}} \begin{cases}>0, & \rho_{n}^{*}=0 \\ =0, & \rho_{n}^{*} \in(0,1) \\ <0, & \rho_{n}^{*}=1 .\end{cases}
$$

The conditions (18) and (19) can be interpreted by noting that, if $p_{n}^{*}$ or $\rho_{n}^{*}$ minimize $L(\mathbf{p}, \boldsymbol{\rho} ; \gamma, \epsilon)$, their partial derivative must vanish unless they lie on some boundary of the feasible set. Conversely, if $p_{n}^{*}$ or $\rho_{n}^{*}$ lie on the boundary of the feasible set, $L(\mathbf{p}, \boldsymbol{\rho} ; \gamma, \epsilon)$ may not decrease by moving to a point in the interior

Solution structure for $p_{n}^{*}$ : Substituting (15) into (18) and solving for $p_{n}^{*}$ yields a closed-form expression for the power allocation

$$
p_{n}^{*}=\rho_{n}\left(\frac{\gamma}{\epsilon}-\frac{1}{\beta_{n}}\right)^{+},
$$

where $(\cdot)^{+}$denotes $\max \{\cdot, 0\}$. For any fixed value of $\rho_{n}$, this is a water filling power allocation. To simplify notation in what follows, it is useful to define the water level $\nu:=\gamma / \epsilon$.
Solution structure for $\rho_{n}^{*}$ : By substituting (15) into (19) and using the optimal power allocation (20) we obtain

$$
\phi_{x}^{\prime}\left(\rho_{n}\right)-\gamma h_{n}(\nu)=0,
$$

where $\phi_{x}^{\prime}\left(\rho_{n}\right)$ is the first derivative of $\phi_{x}\left(\rho_{n}\right)$ with respect to $\rho_{n}$. To simplify notation, we have defined

$$
h_{n}(\nu):=\left[\log \left(\nu \beta_{n}\right)\right]^{+}-\frac{\left(\nu \beta_{n}-1\right)^{+}}{1+\left(\nu \beta_{n}-1\right)^{+}} .
$$

By finding the derivative and solving (21) we obtain a closedform expression for the optimal structure given an idle sensing result $x=0$,

$$
\rho_{n}^{*}=\left\{\begin{array}{ll}
\frac{1}{(\lambda+\mu) T} \log \frac{1}{1-\frac{\lambda+\mu}{\lambda} \gamma h_{n}(\nu)}, & \gamma h_{n}(\nu) \leq \zeta_{0} \\
1, & \text { o.w. }
\end{array},\right.
$$

where $\zeta_{0}=\lambda /(\lambda+\mu)(1-\exp (-(\lambda+\mu) T)$. A similar result is obtained for the busy sensing case, $x=1$,

$$
\rho_{n}^{*}= \begin{cases}0, & \gamma h_{n}(\nu)<\zeta_{1} \\ 1+\frac{\log \left(\frac{\lambda+\mu}{\mu} \gamma h_{n}(\nu)-\frac{\lambda}{\mu}\right)}{(\lambda+\mu) T}, & \zeta_{1} \leq \gamma h_{n}(\nu) \leq 1 \\ 1, & \gamma h_{n}(\nu)>1,\end{cases}
$$

where $\zeta_{1}=\lambda /(\lambda+\mu)(1+\mu / \lambda \exp (-(\lambda+\mu) T)$.

\section{B. Solution algorithm}

The closed-form expressions for $p_{n}^{*}$ and $\rho_{n}^{*}$ depend on the values of the Lagrange multipliers $\gamma$ and $\nu$. In this section we develop a solution algorithm that uses bisection to obtain the optimal values $\gamma^{*}$ and $\nu^{*}$.

The algorithm relies on the monotonicity of the objective function. We first need the following result.

Lemma 3: The function $h_{n}(\nu)$ is non-decreasing in $\nu$.

Proof: The result can be verified by differentiation.

Finding the optimal $\nu^{*}$ given $\gamma$ : We first consider the problem of choosing an optimal value for $\nu$ while keeping $\gamma$ fixed. The monotonicity of $h_{n}(\nu)$ and the closed form expression (23) imply that $\phi_{x}\left(\rho_{n}\right)$ increases monotonically with $\nu$ as well. This property can be used to find the value of $\nu$ which satisfies the rate constraint with equality. In fact, the rate corresponding to an allocation vector $[\mathbf{p}, \rho]$ increases with $\nu$. This can be seen by noting that for fixed $\mathbf{p}$ the achievable rate increases with $\rho$. At the same time, based on (20) it is clear that the allocated power increases as well.

This property can be exploited to find $\nu^{*}(\gamma)$. The algorithm is based on the bisection method and starts by finding upper and lower bounds on the value of $\nu$. We know that an upper bound $\nu_{u}$ exists for any rate constraint $R$, since $\nu \rightarrow \infty$ implies $r \rightarrow \infty$. Similarly, a lower bound $\nu_{l}$ exists since $\nu \rightarrow 0$ implies $r \rightarrow 0$.

Once $\nu_{l}$ and $\nu_{u}$ have been obtained, the bisection method is used to find the value $\nu^{*}(\gamma)$ that satisfies the rate constraint with equality. This corresponds to the inner loop of the flow chart shown in Fig. 3. 
Finding the optimal $\gamma^{*}$ : The above algorithm finds a $\nu^{*}(\gamma)$ which satisfies the rate constraint with equality for a given $\gamma$. However, the power constraint (12) may not be satisfied with equality. To find the corresponding value of $\gamma$, we study the effect of varying $\gamma$ on the objective function and the sum power $\sum_{n} p_{n}$. It is important to study this behavior for the pair of Lagrange multipliers $\left[\gamma, \nu^{*}(\gamma)\right]$ where $\nu^{*}$ is adjusted with $\gamma$ to ensure that the rate constraint remains met at all times.

The slackness condition (16) implies that, at the optimal solution, the rate constraint must be met with equality. We therefore have

$$
\sum_{n} \rho_{n}\left(\gamma, \nu^{*}(\gamma)\right)\left[\log \left(\nu^{*}(\gamma) \beta_{n}\right)\right]^{+}=R,
$$

where $\rho_{n}\left(\gamma, \nu^{*}(\gamma)\right)$ is given by (23) or (24) depending on the sensing result. From this solution structure, it can be seen that decreasing $\gamma$ requires an increase of $\nu$ in order to maintain equality in (25). It can further be shown that decreasing $\gamma$ reduces the objective function (due to the fact that $\log \left(\hat{\nu} \beta_{n}\right) \geq \log \left(\nu \beta_{n}\right)$ for $\hat{\nu} \geq \nu$, decreasing $\gamma$ enables us to reduce $\rho_{n}\left(\gamma, \nu^{*}(\gamma)\right)$ for at least some $\left.n\right)$.

While decreasing $\gamma$ reduces the objective function, we need to find a pair $[\gamma, \nu]$ which satisfies both power and rate constraints. The total allocated sum power, as a function of $\gamma$, is given by

$$
\sum_{n} \rho_{n}\left(\gamma, \nu^{*}(\gamma)\right)\left(\nu^{*}(\gamma)-\frac{1}{\beta_{n}}\right)^{+}
$$

To study the effect of reducing $\gamma$ we can intuitively argue that decreasing $\gamma$ requires increasing $\nu^{*}(\gamma)$. However, since (25) remains constant, (26) increases since the term $\left[\nu^{*}(\gamma)-\frac{1}{\beta_{n}}\right]^{+}$ increases faster than $\left[\log \left(\nu^{*}(\gamma) \beta_{n}\right)\right]^{+}$. Therefore, reducing $\gamma$ results in an allocation with higher sum power. We make this argument rigorous in the following lemma.

Lemma 4: The sum power $\sum_{n} p_{n}$ associated with allocation $\left[\gamma, \nu^{*}(\gamma)\right]$ is a decreasing function in $\gamma$.

Proof: See appendix.

Based on Lemma 4, it is possible to find the optimal value $\gamma^{*}$ in an iterative manner. We know that by decreasing $\gamma$, we keep improving the objective function. However, the total power required for such an allocation grows as $\gamma$ is decreased and, at some point, will exceed $P$. The optimal solution is obtained at this point as illustrated by the fact that all KKT conditions are satisfied.

Computationally, the optimal solution $\left[\gamma^{*}, \nu^{*}\right]$ is again obtained through the bisection method. Similar to before, we first find upper and lower bounds $\gamma_{u}$ and $\gamma_{l}$, respectively. Due to the monotonicity ascertained by Lemma 4 we can then find the optimal solution using the bisection method. This is illustrated by the outer loop of the flow chart shown in Fig. 3.

\section{Average Rate Formulation}

The previous section presented an algorithm for finding the optimal solution to (10)-(14), which minimized the expected overlap between $\mathrm{AH}$ and IS link subject to rate and power

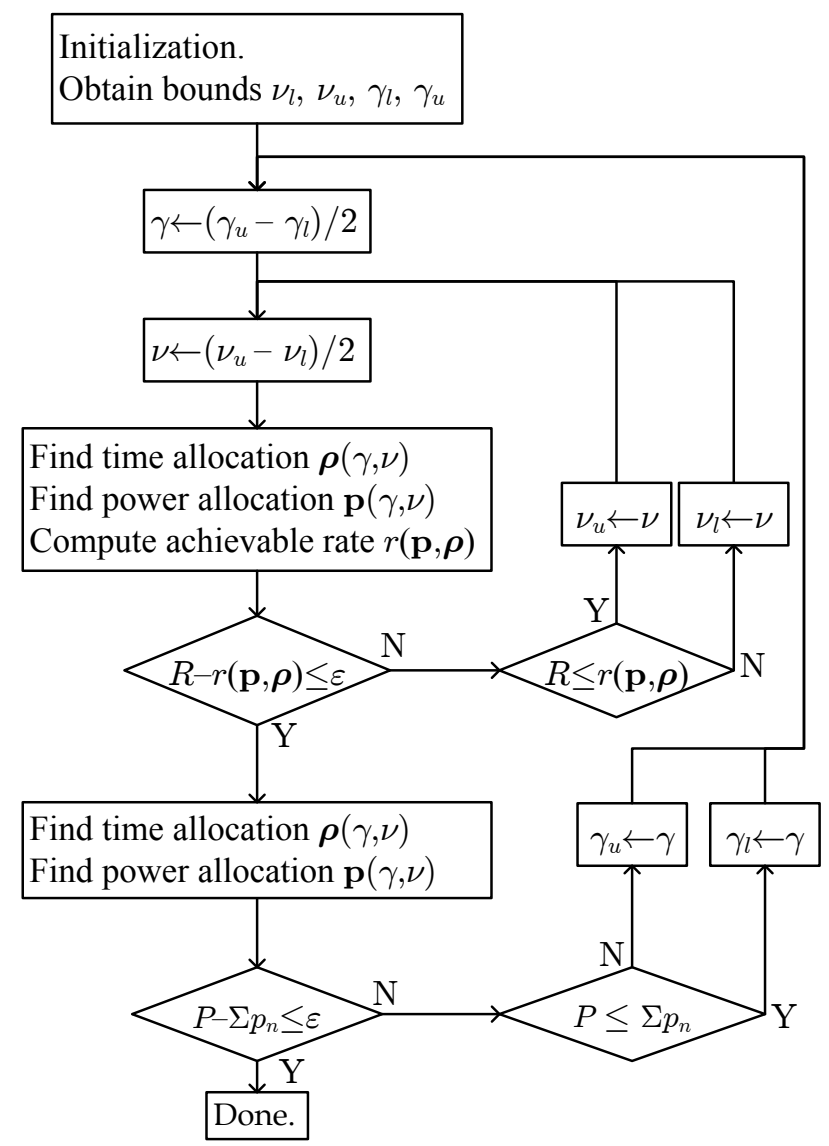

Fig. 3. Flow chart for finding the optimal Lagrange multipliers $\gamma$ and $\nu$ for problem (10)-(14). The inner loop finds $\nu^{*}(\gamma)$ which satisfies the rate constraint (11). The outer loop determines $\gamma^{*}$, which satisfies the power constraint (12)

constraints. The formulation required, however, that the rate constraint was met on a frame-level, allocating the full rate $R$ regardless of $\mathrm{AH}$ network activity.

Many applications do not require the rate constraint to be met on a per-frame level. Instead, it suffices to enforce the constraint in the long-run, averaging over the ON/OFF activity of the $\mathrm{AH}$ link. It is clear that this relaxation has the potential to improve performance significantly, by being more conservative when the sensing outcome is busy, and more aggressive when the outcome is idle.

The average-rate formulation of problem (10)-(14) becomes

$$
\begin{aligned}
\min _{\mathbf{p}_{0}, \mathbf{p}_{1}, \boldsymbol{\rho}_{0}, \boldsymbol{\rho}_{1}} & \sum_{n} \eta_{0} \phi_{0}\left(\rho_{0, n}\right)+\eta_{1} \phi_{1}\left(\rho_{1, n}\right) \\
\text { subject to } & \eta_{0} r\left(\mathbf{p}_{0}, \boldsymbol{\rho}_{0}\right)+\eta_{1} r\left(\mathbf{p}_{1}, \boldsymbol{\rho}_{1}\right) \geq R \\
& \eta_{0} \sum_{n} p_{0, n}+\eta_{1} \sum_{n} p_{1, n} \leq P \\
& \mathbf{p}_{x} \geq 0, \quad x=0,1 \\
& 0 \leq \boldsymbol{\rho}_{x} \leq 1, \quad x=0,1,
\end{aligned}
$$

where $\eta_{0}=\mu /(\lambda+\mu)$ and $\eta_{1}=\lambda /(\lambda+\mu)$ are the stationary distribution probabilities of observing an idle/busy channel, respectively. 
The average problem (27)-(31) is still a convex optimization problem in the variables $\left[\mathbf{p}_{0}, \boldsymbol{\rho}_{0}, \mathbf{p}_{1}, \boldsymbol{\rho}_{1}\right]$ since $\eta_{i} \geq 0$. Twice as many decision variables are necessary because we obtain different allocations depending on the sensing outcome. Nevertheless, the solution structure developed in Sec. III still applies since, by taking derivatives with respect to the decision variables, the problem nicely decouples. Furthermore, the solution algorithm presented in the previous section can be extended to the average case in a straightforward manner.

\section{NUMERICAL RESUltS}

This section presents numerical performance results for the power and timing allocation proposed in this paper. The simulation setup corresponds to Fig. 1 and consists of a single IS link with $N=5$ sub-channels and frame duration $T=1 \mathrm{~s}$. The AH system overlaps with all IS sub-channels and evolves according to a CTMC with parameters $\lambda=1 \mathrm{~s}^{-1}$ and $\mu=1 \mathrm{~s}^{-1}$. All results are shown for the average-rate case (27)-(31).

The IS channel is assumed to be flat Rayleigh fading and for simplicity we assume that all sub-channels fade independently with identical distribution. Further, we assume that the channel varies slowly with respect to the evolution of the CTMC and consider a block fading model. It is assumed that through some feedback mechanism, the channel $\beta_{n}$ is known to the transmitter at the beginning of every frame (as frequently assumed in OFDMA systems).

The performance of the proposed method is compared with a reference scheme that only performs power but no timing allocation. Specifically, the reference scheme solves the following optimization problem

$$
\begin{aligned}
\underset{\mathbf{p}}{\min _{n}} & \sum_{n} p_{n} \\
\text { s.t. } & \sum_{n} \log \left(1+\beta_{n} p_{n}\right) \geq R \\
& \sum_{n} p_{n} \leq P \\
& p_{n} \geq 0, \quad \forall n .
\end{aligned}
$$

It is well-known that the above optimization problem admits a water filling solution [21]. In order to compare the expected transmission time overlaps, we assume that if a sub-channel is allocated positive power $p_{n}>0$ under the reference scheme, this sub-channel will be used for the entire frame duration. Naturally, sub-channels that are not allocated any power also have zero transmission time.

\section{A. Average transmission overlap}

The average transmission overlap between IS and $\mathrm{AH}$ link (normalized by the frame duration) is shown in Fig. 4 for varying IS rates. At low rates $R$ there is much flexibility in allocating resources which is effectively used by the interference-aware scheme to suppress interference. The reference scheme, on the other hand, uses sub-channels for the entire frame duration. The average overlap is therefore dominated by the AH links steady-state behavior (the average

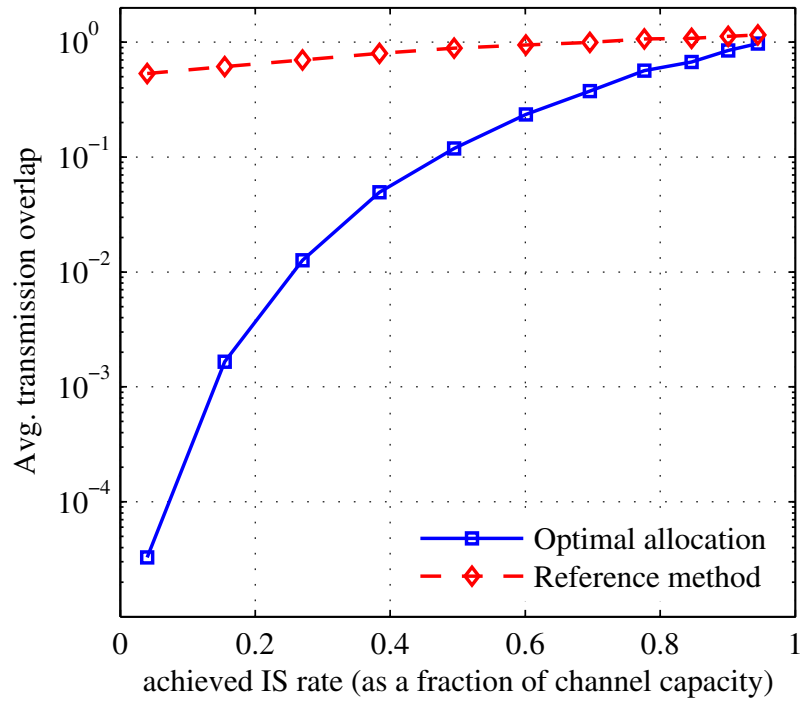

Fig. 4. Average time overlap between both systems. The interference-aware resource allocation reduces interference significantly for low IS rates.

overlap only slightly increases since for larger $R$, more subchannels need to be used).

The performance curves in Fig. 4 are plotted on a logarithmic scale, which demonstrates that for low rate constraints the overlap between both systems can be reduced effectively.

\section{B. Average interference metric}

In Fig. 5 both schemes are compared based on the average expected interference overlap $\mathbb{E}\left[\sum_{n} p_{n} / \rho_{n} \phi_{x}\left(\rho_{n}\right)\right]$. This corresponds to the metric that has been introduced in the optimization problem (5)-(9). Although, the interference-aware method does not optimize for this metric, we observe similar trends compared to the plots of the transmission time overlap. For low rate constraints, interference is again reduced. At high rates, the performance curves converge, as the IS link has less flexibility to assign resources in an interference-aware fashion.

\section{CONCLUSION}

In conclusion, this paper has introduced a cognitive coexistence framework between infrastructure and ad-hoc networks. Based on sensing and predicting the AH link's activity, the IS system allocated power and transmission time such as to minimize the expected transmission overlap between both systems. In this way, we derived an interference-aware resource allocation method that reduced interference effectively. Based on the mathematical framework of convex programming, we found closed-form solutions and derived an iterative algorithm for finding the optimal values of the Lagrange multipliers. Finally, we extended our results to the average-rate scenario, in which power and rate constraints are met on average rather than on a frame-by-frame basis. The additional flexibility of allocating both across frequency and time enables further interference reduction. 


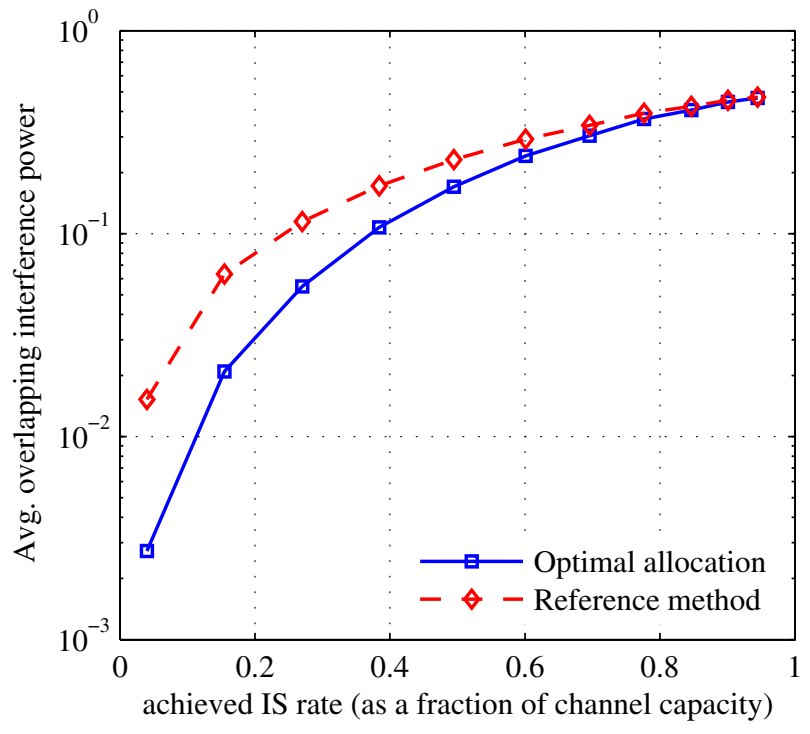

Fig. 5. Average overlapping interference power $\mathbb{E}\left[\sum_{n} p_{n} / \rho_{n} \phi_{x}\left(\rho_{n}\right)\right]$ The interference-aware scheme outperforms the reference based on this metric as well.

\section{APPENDIX \\ PROOF OF LEMMA 4}

The proof proceeds in two steps: we first show that decreasing $\gamma$ leads to a reduced value of the objective function and then use the result to prove that this implies a higher sum power.

Part 1: Consider a pair $[\gamma, \nu]$ which satisfies the rate constraint

$$
\sum_{n} \rho_{n}(\gamma, \nu)\left[\log \left(\nu \beta_{n}\right)\right]^{+}=R
$$

where $\rho_{n}(\gamma, \nu)$ is given by (23) or (24) (depending on the sensing result). Decreasing $\gamma$ to $\bar{\gamma}<\gamma$ and imposing

$$
\sum_{n} \rho_{n}(\bar{\gamma}, \bar{\nu})\left[\log \left(\bar{\nu} \beta_{n}\right)\right]^{+}=R
$$

leads to a new pair $[\bar{\gamma}, \bar{\nu}]$ for which $\bar{\nu}>\nu$. Since $\left[\log \left(\bar{\nu} \beta_{n}\right)\right]^{+} \geq\left[\log \left(\nu \beta_{n}\right)\right]^{+}, \rho_{n}(\bar{\gamma}, \bar{\nu})<\rho_{n}(\gamma, \nu)$ for some $n$. Due to the monotonicity and convexity of (3) and (4), this leads to a decrease in the objective value

$$
f(\boldsymbol{\rho}(\bar{\gamma}, \bar{\nu})):=\sum_{n} \phi_{x}\left(\rho_{n}(\bar{\gamma}, \bar{\nu})\right) .
$$

Part 2: Define $p_{n}(\gamma, \nu)$ as the optimal power allocation given the pair $[\gamma, \nu]$. We now show that $\sum_{n} p_{n}(\bar{\gamma}, \bar{\nu}) \geq$ $\sum_{n} p_{n}(\gamma, \nu)$, i.e., reducing $\gamma$ leads to a larger sum power allocation. Arguing by contradiction, we assume $\sum_{n} p_{n}(\gamma, \nu)>$ $\sum_{n} p_{n}(\bar{\gamma}, \bar{\nu})$. But then, the fact that both $[\gamma, \nu]$ and $[\bar{\gamma}, \bar{\nu}]$ achieve the same rate, and the result from Part 1 imply that $[\gamma, \nu]$ is not an optimal solution for rate constraint $R$ and power constraint $\sum_{n} p_{n}(\gamma, \nu)$, which is a contradiction.

\section{REFERENCES}

[1] Q. Zhao and B. M. Sadler, "Dynamic Spectrum Access: Signal Processing, Networking, and Regulatory Policy," IEEE Signal Process. Mag., vol. 55, no. 5, pp. 2294-2309, May 2007.

[2] I. Akyildiz, W. Lee, M. Vuran, and S. Mohanty, "NeXt generation/dynamic spectrum access/cognitive radio wireless networks: A survey," Computer Networks, vol. 50, no. 13, pp. 2127-2159, Sep. 2006.

[3] J. L. Burbank and W. T. Kasch, "IEEE 802.16 Broadband Wireless Technology and Its Application to the Military Problem Space," in Proc. IEEE Military Communications Conference (MILCOM), vol. 3, Oct. 2000, pp. 1905-1911.

[4] L. Berlemann, C. Hoymann, G. Hiertz, and B. Walke, "Unlicensed Operation of IEEE 802.16: Coexistence with 802.11(A) in Shared Frequency Bands," in Proc. IEEE International Symposium on Personal, Indoor and Mobile Radio Communications, Sep. 2006.

[5] X. Fu, W. Ma, and Q. Zhang, "The IEEE 802.16 and 802.11a Coexistence in the License-Exempt Band," in Proc. IEEE WCNC, Mar. 2007, pp. 1944-1949.

[6] H.-y. Wei and R. Gitlin, "Two-hop-relay architecture for next-generation WWAN/WLAN integration," IEEE Wireless Communications, vol. 11, no. 2, pp. 24-30, Apr. 2004.

[7] I. Wong and B. Evans, Resource Allocation in Multiuser Multicarrier Wireless Systems. Springer Science+Business, 2008.

[8] K. Kim, Y. Han, and S.-L. Kim, "Joint Subcarrier and Power Allocation in Uplink OFDMA Systems," IEEE Commun. Lett., vol. 9, no. 6, pp. 526-528, Jun. 2005.

[9] C. Y. Wong, R. S. Cheng, K. B. Letaief, and R. D. Murch, "Multiuser OFDM with Adaptive Subcarrier, Bit, and Power Allocation," IEEE J. Sel. Areas Commun., vol. 17, no. 10, pp. 1747-1758, Oct. 1999.

[10] T. Peng, W. Wang, Q. Lu, and W. Wang, "Subcarrier Allocation Based on Water-filling Level in OFDMA-based Cognitive Radio Networks," in Proc. ACM International Conference on Wireless Communications, Networking and Mobile Computing, Sep. 2007, pp. 196-199.

[11] P. Wang, M. Zhao, L. Xiao, S. Zhou, and J. Wang, "Power Allocation in OFDM-based Cognitive Radio Systems," in Proc. IEEE Global Communications Conference (Globecom), Nov. 2007, pp. 4061-4065.

[12] J. Acharya and R. D. Yates, "A Framework for Dynamic Spectrum Sharing between Cognitive Radios," in Proc. IEEE International Communications Conference (ICC), Jun. 2007, pp. 5166-5171.

[13] A. T. Hoang and Y.-C. Liang, "A Two-Phase Channel and Power Allocation Scheme for Cognitive Radio Networks," in Proc. IEEE International Symposium on Personal, Indoor and Mobile Radio Communications, Sep. 2006.

[14] W. Wang and X. Liu, "List-coloring based channel allocation for open-spectrum wireless networks," in Proc. IEEE Vehicular Technology Conference (VTC), Sep. 2005, pp. 690-694.

[15] H. Zheng and C. Peng, "Collaboration and fairness in opportunistic spectrum access," in Proc. IEEE International Conference on Communications (ICC), May 2005, pp. 3132-3136.

[16] S. Geirhofer, L. Tong, and B. M. Sadler, "A Cognitive Framework for Improving Coexistence Among Heterogeneous Wireless Networks," in Proc. IEEE Global Communications Conference (Globecom), Nov. 2008.

[17] — "Dynamic Spectrum Access in the Time Domain: Modeling and Exploiting Whitespace," IEEE Commun. Mag., vol. 45, no. 5, pp. 66-72, May 2007.

[18] — "Cognitive Medium Access: Constraining Interference Based on Experimental Models," IEEE J. Sel. Areas Commun., vol. 26, no. 1, pp. 95-105, Jan. 2008.

[19] X. Qiu and K. Chawla, "On the Performance of Adaptive Modulation in Cellular Systems," IEEE Trans. Commun., vol. 47, no. 6, pp. 884-895, Jun. 1999.

[20] S. I. Resnick, Adventures in Stochastic Processes. Birkhäuser, 1992.

[21] S. Boyd and L. Vandenberghe, Convex Optimization. Cambridge University Press, 2004. 